\title{
Geriatric oncology: questions, answers and guidelines
}

\author{
Matthias Unseld · Christine Marosi (iD)
}

Received: 17 April 2020 / Accepted: 18 May 2020 / Published online: 9 June 2020

(C) The Author(s) 2020

\begin{abstract}
Summary
Background With the aging of populations, the number of elderly persons with cancer will increase. Due to the high individuality of elderly persons and their varying patterns of resources and disabilities, cancer treatment for elderly cancer patients needs to be individually adapted. To achieve this, geriatric medicine has established the comprehensive geriatric assessment (CGA). This short review presents the evidence of feasibility and impact of CGA on cancer treatment in elderly patients, as recommended by the American Society of Clinical Oncology (ASCO) in 2018.

Methods A systematic review of the literature and a Delphi Consensus with a panel of experts cooperated to compile the evidence for choosing the most adequate treatment for elderly cancer patients.

Results There is evidence that CGA makes it possible to predict the occurrence of complications of chemotherapy and of health deterioration, as well as death within 1 year.

Conclusion The ASCO has recognized the optimization of cancer therapy for elderly patients as a priority.

Keywords Tumor treatment for elderly · Individual resources and restrictions - Functional indepence · Chosing endpoints for clinical trials · Non-cancerspecific life expectancy
\end{abstract}

\section{Unseld, MD, PhD · C. Marosi, MD ( $\varangle)$}

Clinical Division of Palliative Care, Department for Internal

Medicine I, Medical University of Vienna, Währinger

Gürtel 18-20, 1090 Vienna, Austria

christine.marosi@meduniwien.ac.at

M. Unseld, MD, PhD

matthias.unseld@meduniwien.ac.at

\section{Introduction}

Raising awareness of the necessity to adapt cancer therapy to the individual needs and resources of elderly cancer patients is the mission for geriatric oncology in the 21st century. Since its beginnings around the turn of the millennium [1], geriatric oncology has provided tools for the assessment of functioning and vulnerabilities in older patients undergoing cancer therapies. The International Society of Geriatric Oncology (SIOG) has advocated for many years that older adults undergo a geriatric assessment (GA) before the start of cancer treatment and that possible findings of this assessment are taken into account to potentially adapt and assist this therapy. In a seminal paper, Hamaker et al. showed that this practice made it possible to detect a previously unknown geriatric syndrome in half of the patients tested and that geriatric counselling led to an increase in the therapy planned in the same number of patients as to an alleviation of therapy [2]. Meanwhile, a lot of experience has been gained by using and optimizing the assessment tools, as well by evaluating their impact in clinical trials.

Although more than two thirds of cancers develop in individuals aged 65 years or more [3], older patients have been underrepresented in clinical trials for decades, first due to age limits, and later due to selective inclusion and exclusion criteria hindering trial participation of older subjects with age-related comorbidities. This exclusion has led to a situation in which evidence-based treatment standards are lacking for older patients with cancer, despite this group representing the majority of cancer patients. In the US, the Institute of Medicine acknowledged this fundamental gap in cancer care and implemented actions for change [4]. 
Supriya Mohile coordinated a panel of experts of different disciplines that reviewed the literature for the development of an American Society of Clinical Oncology (ASCO) guideline for geriatric oncology published in August 2018 in the Journal of Clinical Oncology [5]. Their duty was to evaluate the feasibility and efficacy of the different tests of a comprehensive geriatric assessment (CGA) to guide the management of vulnerabilities in older adult patients receiving chemotherapy.

The ASCO guideline provides answers to four questions:

1. Should GA be used in older adults with cancer to predict adverse outcomes from chemotherapy?

2. For older patients that are considering undergoing chemotherapy, which GA tools should clinicians use to predict adverse outcomes (including chemotherapy toxicity and mortality)?

3. What general non-cancer-specific life expectancy data for community-dwelling patients should clinicians consider to estimate and best inform treatment decision-making for older patients with cancer?

4. How should GA be used to guide the management of older patients with cancer?

This guideline was developed by a multidisciplinary expert panel and is conceived as a snapshot of the state of the art as of August 2018. It is not updated und thus open for progress. The authors claim neither completeness nor exclusivity. They emphasize that new evidence may emerge and should be taken into account, as well as the fact that the guideline is not intended as a substitute for the individual assessment of a given patient. The recommendations are based on the literature available at the time of writing. The authors compiled data from 68 studies, including 17 randomized controlled trials (RCTs).

These guidelines will now be briefly summarized and the subjectively most substantial references discussed with regard to their respective topics; nevertheless, studying the guidelines in full text is strongly recommended.

\section{Question 1: Should geriatric assessment be used in older adults with cancer to predict adverse outcomes from chemotherapy?}

Recommendation 1 CGA should be used in patients aged 65 years and older in order to identify vulnerabilities or geriatric impairments that are not routinely captured in oncology assessments.

Strength of recommendation: strong

This recommendation is based on literature showing that CGA detects problems that are not routinely detected by routine history taking, physical examination and evaluation of the Karnovsky or Eastern Cooperative Oncology Group (ECOG) performance scores.
A CGA is a multidimensional assessment evaluating physical performance, functional status including activities of daily living and instrumental activities of daily living (ADL and IADL, respectively), history of falls, comorbidities, depression, cognition, social activities and social support as well as nutritional status. There are some validated short screening tools, like the G8 [6] and the Vulnerable Elders Survey (VES-13) [7], which are able to predict mortality within 1 and 2 years, respectively, but unable to provide the information of a full assessment.

There is already strong evidence that a CGA is able to identify older individuals at increased risk of mortality. One of the most convincing studies on this topic is the one conducted by Aaldricks et al., describing a three-item Geriatric Prognostic Index [8]:

- Decreased food intake in the preceding 3 months

- Dependence in terms of shopping

- Use of more than three medications

The score is associated with an increased risk of mortality of 1.58, 2.32 and 5.58 for one, two or three items, respectively. The association between abnormal findings in CGA and mortality has been confirmed by many studies in geriatric oncology.

For the identification of patients at increased risk for chemotherapy toxicity, two composite test tools have already been validated:

- The Chemotoxicity Calculator by the Cancer and Aging Research Group (CARG) led by Arti Hurria [9] combines standard clinical data with GA data on prior falls, ability to walk one block, hearing problems, social support and IADL. This tool takes less than $5 \mathrm{~min}$ to complete and is freely available at: www.mycarg.org/Chemo_Toxicity_Calculator.

- The CRASH score (Chemotherapy Risk Assessment Scale for High-Age Patients) was designed by evaluating prospectively recorded toxicity data of 585 patients older than 70 years, treated at the Moffitt Affiliate Research Network [10]. It provides estimates for both hematologic toxicity $\geq 3$ and severe non-hematologic toxicity. The CRASH score contains several elements of CGA such as IADL, Mini Mental State Examination (MMSE) [11] and Mini Nutritional Assessment (MNA) [12, 13]. The CRASH score takes $30 \mathrm{~min}$ to complete and is also freely available at: https://moffitt.org/for-healthcareproviders/clinical-programs-and-services/senioradult-oncology-program-tools.

Furthermore, CGA enables a prediction of completion of chemotherapy, the risk of hospitalization and the risk of functional decline. 
Question 2: For older patients that are considering undergoing chemotherapy, which GA tools should clinicians use to predict adverse outcomes?

Recommendation 2 At a minimum, assessment of function, comorbidities, falls, depression, cognition and nutrition, ideally with the validated tools.

Strength of recommendation: high

Strength for each tool alone: moderate

In order to avoid repetitions, the above-mentioned tools, such as ADL/IADL, G8 or VES-13, CARG Score or CRASH Score, MNA, and MMSE, are not presented again. In addition to a complete CGA, a question on falls during the preceding 6 months, a question on living situation (alone or with someone else), an evaluation of co-morbidities with the Cumulative Illness Rating Scale for geropsychiatric patients (CIRSG) [14] or Charlson Comorbidity score [15], screening for depression with the Geriatric Depression Scale [16], screening for impaired cognition with the MiniCog $[17,18]$ instead of MMSE and evaluation of physical function with the Timed Up and Go Test (TUG) [19] are suggested.

For the quantification of comorbidities, the CIRS-G is available online at https://www.mdcalc.com/cumu lative-illness-rating-scale-geriatric-cirs-g, whereas the less detailed Charlson Co-morbidity Index is available at: https://www.mdcalc.com/charlson-comorbidityindex-cci. The geriatric depression scale is a 15item self-administered questionnaire that can usually be completed in less than 5 min (Sheik) and available in German at https://www.geriatrie-bochum.de/ assessment/geriatrische-depressions-skala-gds.php.

The Mini-Cogc test can be administered in less than 3 min and consists of a three-item recall and a clockdrawing test (https://mini-cog.com/) and is easier to perform than the MMSE but as informative. The TUG measures the time required for getting up from a chair with armrests, going $3 \mathrm{~m}$, turning, going back to the chair and sitting down. This simple test provides information on a person's mobility, balance and risk of falls and is completed within a few seconds. There are cut-off values for different age groups.

It has been repeatedly shown that the majority of these tests can be done as self-administered questionnaires and that patients are able to complete them either themselves or with assistance from their proxies during the waiting time in an outpatient unit. Using hand-held touch screen computers is reliable and often preferred [20]. Of course, functional tests such as the TUG and the screening for cognitive function need to be administered by trained personnel. This can be achieved in less than 15 min.
Question 3: What general non-cancer-specific life expectancy data for community-dwelling patients should clinicians consider to estimate and best inform treatment decision-making for older patients with cancer?

Recommendation 3 This question on the probability of survival for the next 5-10 years can be answered using validated tools established for large cohorts of US citizens aged $50+$ or $65+$ after 2000 , e.g. the Lee Schonberg Index [21, 22], available at https:// eprognosis.ucsf.edu/leeschonberg.php.

Of note, to determine the "non-cancer" probability of death, the question on "presence of cancer" needs to be answered with "no". The prognosis tools rely on data such as age, sex, performance status, mastering of ADLs and IADLs, comorbidities, life style factors and self-reported health.

\section{Strength of recommendation: strong}

In a next step, the probability of survival with optimal cancer treatment needs to be estimated. Considering both results allows the best counselling for patients and potentially draws attention to other health conditions that should be taken into account, regardless of whether anti-cancer treatment is initiated or not.

Question 4: How should GA be used to guide the management of older patients with cancer?

Recommendation 4 There are two parts to this recommendation: The first is that a GA will impact treatment decisions, e.g. in choosing therapeutic regimens with a low burden of side effects and in adapting dosage of chemotherapies to the individual organ functions of the patients, while respecting the guidelines for the application of growth factors for elderly patients. The second aspect is to consider interventions able to address the vulnerabilities identified by a GA in order to improve the condition of elderly persons.

In fact, there are no completed RCTs to prove that GA-guided therapies improve the outcomes of elderly cancer patients. However, there is already wide experience that GA-guided interventions improve the outcomes of many non-cancer issues.

A Delphi consensus was elaborated by the panel that the two recommendations outlined above should be implemented in cancer care for the elderly. In Europe as well as in the US and Canada, several RCTs are currently underway to assess the feasibility and impact on the outcomes of elderly cancer patients. The endpoints of the studies are mostly composite endpoints, focusing on duration of survival and duration of progression free survival, quality of life, rates of severe chemotherapeutic toxicity, rate of hospitalization, health care utilization, change in functional status, as well as change in psychological status and caregiver quality of life and caregiver satisfaction. 
Strength of the recommendation: as long as the results of these trials are not available, moderate

The implementation of GA-guided interventions necessitates rethinking the workflow of therapeutic decision-making for elderly cancer patients. On the other hand, it is anticipated that integrating GA and GA-driven interventions prevents chemotherapeutic toxicities, avoidable infectious complications and complications related to functional decline, meaning that the expenditure of time and resources used for the GA is amply compensated.

This ASCO guideline was released in July 2018 and is continuously implemented in the US.

The numbers of patients with cancer aged 65 years or older are going to increase rapidly over the coming years. Austrian oncologists should prepare for this challenge in an informed and considered manner.

\section{Take home message}

- The numbers of elderly patients with cancer will increase rapidly

- Planning cancer therapy for elderly cancer patients should take into account CGA and geriatric interventions if needed

- CGA makes it possible to predict severe toxicity of chemotherapy

- CGA enables the prediction of death within 1 year

- CGA makes it possible to predict early deterioration of health status

Funding Open access funding provided by Medical University of Vienna.

Conflict of interest M. Unseld and C. Marosi declare that they have no competing interests.

Open Access This article is licensed under a Creative Commons Attribution 4.0 International License, which permits use, sharing, adaptation, distribution and reproduction in any medium or format, as long as you give appropriate credit to the original author(s) and the source, provide a link to the Creative Commons licence, and indicate if changes were made. The images or other third party material in this article are included in the article's Creative Commons licence, unless indicated otherwise in a credit line to the material. If material is not included in the article's Creative Commons licence and your intended use is not permitted by statutory regulation or exceeds the permitted use, you will need to obtain permission directly from the copyright holder. To view a copy of this licence, visit http://creativecommons.org/licenses/by/4.0/.

\section{References}

1. Monfardini S, Balducci L. A comprehensive geriatric assessment (CGA) is necessary for the study and the management of cancer in the elderly. Eur J Cancer. 1999;35(13):1771-2.

2. Hamaker ME, Jonker JM, de Rooij SE, Vos AG, Smorenburg $\mathrm{CH}$, van Munster BC. Frailty screening methods for predicting outcome of a comprehensive geriatric assessment in elderly patients with cancer: a systematic review. LancetOncol. 2012;13(10):e437-e44.
3. Smith BD, Smith GL, Hurria A, Hortobagyi GN, Buchholz TA. Future of cancer incidence in the United States: burdens upon an aging, changing nation. J Clin Oncol. 2009;27(17):2758-65.

4. Levit LBE, Nass S, Ganz PA. Delivering high-quality cancer care: charting a new course for a systemin crisis. Committee on improving the quality of cancer care: addressing the challenges of an aging population. Washington: Institute of Medicine of the National Academies; 2013. Board on Health Care Services.

5. Mohile SG, Dale W, Somerfield MR, Hurria A. Practical assessment and management of vulnerabilities in older patients receiving chemotherapy: ASCOguidelineforgeriatric oncology summary. J Oncol Pract. 2018;14(7):442-6.

6. Bellera C, Soubeyran P. [How to identify frailty in older patients with cancer? Available tools]. Comment identifier les personnes agees fragiles? Outils a disposition. Cancer Radiother. 2015;19(6-7):382-5.

7. Min L, Ubhayakar N, Saliba D, Kelley-Quon L, Morley E, Hiatt J, et al. The vulnerable elders survey-13 predicts hospital complications and mortality in older adults with traumatic injury: a pilot study. JAm Geriatr Soc. 2011;59(8):1471-6.

8. AaldriksAA, MaartenseE, NortierHJWR, vanderGeestLGM, le Cessie S, Tanis BC, et al. Prognostic factors for the feasibility of chemotherapy and the Geriatric Prognostic Index (GPI) as risk profile for mortality before chemotherapy in the elderly. Acta Oncol. 2016;55(1):15-23.

9. Hurria A, Togawa K, Mohile SG, Owusu C, Klepin HD, Gross CP, et al. Predicting chemotherapy toxicity in older adults with cancer: a prospective multicenter study. J Clin Oncol. 2011;29(25):3457-65.

10. Extermann M, Boler I, Reich RR, Lyman GH, Brown RH, DeFelice J, et al. Predicting the risk of chemotherapy toxicity in older patients: the Chemotherapy Risk Assessment Scale for High-Age Patients (CRASH) score. Cancer. 2012;118(13):3377-86.

11. Folstein MF, Folstein SE, McHugh PR. "Mini-mental state”. A practical method for grading the cognitive state of patients for the clinician. J Psychiatr Res. 1975;12(3):189-98.

12. Guigoz Y, Vellas B, Garry PJ. Assessing the nutritional status of the elderly: the Mini Nutritional Assessment as part of the geriatric evaluation. Nutr Rev. 1996;54(1 Pt2):S59-S65.

13. Kaiser MJ, Bauer JM, Ramsch C, Uter W, Guigoz Y, Cederholm T, et al. Validation of the Mini Nutritional Assessment short-form (MNA-SF): a practical tool for identification of nutritional status. J Nutr Health Aging. 2009;13(9):782-8.

14. Miller MD, Paradis CF, Houck PR, Mazumdar S, Stack JA, Rifai $\mathrm{AH}$, et al. Rating chronic medical illness burden in geropsychiatric practice and research: application of the Cumulative Illness Rating Scale. Psychiatry Res. 1992;41(3):237-48.

15. Charlson ME, Pompei P, Ales KL, MacKenzie CR. A new method of classifying prognostic comorbidity in longitudinal studies: development and validation. J Chronic Dis. 1987;40(5):373-83.

16. Yesavage JA, Brink TL, Rose TL, Lum O, Huang V, Adey M, et al. Development and validation of a geriatric depression screening scale: a preliminary report. J Psychiatr Res. 1982;17(1):37-49.

17. Borson S, Scanlan J, Brush M, Vitaliano P, Dokmak A. The mini-cog: a cognitive 'vital signs' measure for dementia screening in multi-lingual elderly. Int J Geriatr Psychiatry. 2000;15(11):1021-7.

18. Borson S, Brush M, Gil E, Scanlan J, Vitaliano P, Chen J, et al. The Clock Drawing Test: utility for dementia detection in multiethnic elders. J Gerontol A Biol Sci Med Sci. 1999;54(11):M534-M40. 
19. Podsiadlo D, Richardson S. The timed "Up \& Go": a test of basic functional mobility for frail elderly persons. J Am Geriatr Soc. 1991;39(2):142-8.

20. Hurria A, Akiba C, Kim J, Mitani D, Loscalzo M, Katheria V, et al. Reliability, validity, and feasibility of a computer-based geriatric assessment for older adults with cancer. J Oncol Pract. 2016;12(12):e1025-e34.

21. Yourman LC, Lee SJ, Schonberg MA, Widera EW, Smith AK. Prognostic indices for older adults: a systematic review. JAMA. 2012;307(2):182-92.

22. Suemoto CK, Ueda P, Beltran-Sanchez H, Lebrao ML, Duarte YA, Wong R, et al. Development and validation of a 10-year mortality prediction model: meta-analysis of individual participant data from five cohorts of older adults in developed and developing countries. J GerontolABiol Sci Med Sci. 2017;72(3):410-6.
Publisher's Note Springer Nature remains neutral with regard to jurisdictional claims in published maps and institutional affiliations.

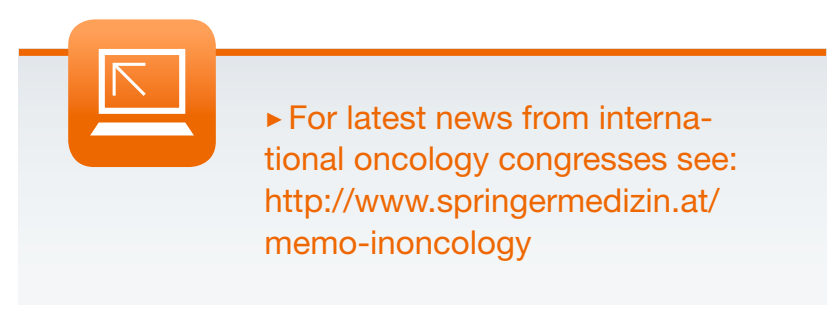

